\title{
Cloud chambers and crystal growth: Effects of electrically enhanced diffusion on dendrite formation from neutral molecules
}

\author{
Kenneth G. Libbrecht* and Victoria M. Tanusheva \\ Norman Bridge Laboratory of Physics, California Institute of Technology 264-33, Pasadena, California 91125
}

(Received 14 October 1998)

\begin{abstract}
We present an extension of the solvability theory for free dendrite growth that includes the effects of electrically enhanced diffusion of neutral polar molecules. Our theory reveals a new instability mechanism in free dendrite growth, which arises when electrically enhanced diffusion near the dendrite tip overwhelms the stabilizing influence of surface tension. This phenomenon is closely related to the growth instability responsible for the visualization of charged particle tracks in cloud chambers, and is expected for enhanced diffusion of neutral molecules, but not for the case of ionic diffusion. Above a threshold applied potential, the crystal growth can no longer be described by the usual solvability theory, and requires a new physical mechanism to limit the growth velocity. We also describe experimental observations of the free dendrite growth of ice crystals from water vapor in supersaturated normal air. These observations demonstrate the calculated growth instability, which results in the rapid growth of branchless ice needles with a tip velocity 5-50 times the normal dendrite tip velocity. The production of clean ice needles is useful for the study of ice crystal growth from vapor, allowing the controlled growth of isolated single-crystal samples. This instability mechanism may find further application in crystal growth from a wide variety of polar molecules. [S1063-651X(99)12203-7]
\end{abstract}

PACS number(s): 68.70. + w, 81.30.Fb

\section{INTRODUCTION}

The formation of stable spatial patterns is a fundamental problem in the study of nonlinear nonequilibrium systems [1-4]. A now-standard example of a simple pattern-forming system is the diffusion-limited growth of free crystalline dendrites, which are nearly ubiquitous products of solidification [5]. Dendrite growth often (but not always) results when diffusion regulates the propagation of a solidification front through a metastable medium, as occurs during solidification controlled by thermal diffusion in an overcooled melt, and solidification via particle diffusion of a supersaturated gas in a solvent vapor. Dendritic solidification is found frequently in nature, for example, the formation of snow crystals in the atmosphere, dendritic patterns in rocks, and is also often seen in the formation of metal castings, weldments, and other metallurgical processes [5].

For the work presented here we will mainly be concerned with the simplest type of stable dendrite growth, in which surface kinetics and surface transport processes are considered negligible. Such systems have now been extensively studied both experimentally and theoretically in a variety of circumstances over several decades [1-3]. In this simple case the dendrite attributes include (i) a nearly parabolic dendrite tip, which advances with constant velocity in a shapepreserving growth morphology; and (ii) secondary and higher-order sidebranches, which form behind the growing tip.

Microscopic solvability theory has been very successful in furnishing a mathematically consistent and dynamically stable solution for this simple dendrite growth [6-10], in-

*URL: http://www.its.caltech.edu/ atomic/. Electronic address: kgl@caltech.edu cluding essentially just two physical processes: diffusion and surface tension. The theory has been widely tested, and achieves good quantitative agreement with a number of numerical and experimental systems. In particular, the theory was recently extended to the fully three-dimensional case [10], comparing well with experimental observations and numerical simulations in most cases $[11,12]$.

It was found over a half-century ago that the diffusion equation allows the solution of a parabolic solidification front which advances at a constant velocity, known as the Ivantsov solution [3,13]. The Ivantsov dendrite is parametrized by the radius of curvature of its tip, $R$, and the tip growth velocity, $v$, which are related by $v R=2 D P(\Delta)$, where $D$ is the diffusion constant and the dimensionless Peclet number $P$ is found to be a function of the generalized undercooling or supersaturation. In the limit of small $P$, or equivalently small $v, P$ is determined by the relationship $\Delta$ $=-P \ln P$.

The diffusion equation alone provides only this relation between $R$ and $v$, however, which is insufficient to determine either. Furthermore, the Ivantsov solution was found to be unstable via the Mullins-Sekerka instability [14]. Because smaller $R$ implies faster $v$, this instability tends to decrease the radius of curvature of a growing dendrite tip with time, in contrast to the observed shape-preserving growth morphology.

Surface tension, via the Gibbs-Thomson effect, provides the mechanism which competes with the Mullins-Sekerka instability and produces stable dendritic growth. During the development of solvability theory it was realized that surface tension introduces a singular perturbation into the problem, which is difficult to deal with mathematically [6-10]. As was found in early computer simulations, this leads to the result that stable dendrite growth requires an anisotropic surface tension, since otherwise the dendrite solution is unstable 
to a tip-splitting perturbation. Including an anisotropic surface tension, solvability theory provides a solution which in three dimensions (3D) is nearly a paraboloid of revolution in the vicinity of the dendrite tip, growing at constant tip velocity $[10,11]$. Instabilities and noise amplification producing sidebranching have also been well studied, and it has been found that sidebranch formation has a minimal effect on the tip growth behavior, and can typically be ignored without significant consequence in the calculation of $R$ and $v$.

In the present paper we focus on simple dendrite growth from a supersaturated vapor in a solute gas, and assume the solute molecules are neutral but possess a significant electric polarizability. By applying an electrical potential to the growing dendrite we then produce enhanced diffusion of the solute molecules which can greatly perturb the normal dendrite growth. This is a specific case of what can be considered a general class of externally forced solidification problems, namely dendrite growth in the presence of external force terms in the diffusion equation, especially when the external forces depend on the presence of the dendrite itself.

\section{Electric field effects}

There have been numerous studies of pattern formation induced by electrically enhanced particle mobility, both in charged and uncharged systems. A favorite experimental system is electrodeposition, which is typically controlled by the diffusion and migration of ions in an electrolytic medium between two electrodes [4]. These systems often produce a fractal-like growth resulting from diffusion-limited aggregation, but a wide variety of growth morphologies have been observed, including simple dendritic patterns [15]. In addition to electrically enhanced diffusion and surface tension, however, these systems are governed by a number of complex factors, including activation chemistry and transport dynamics near the electrode surfaces, and much work has gone into understanding the interplay of the different electrochemical and physical processes [16].

By contrast, the system under consideration here is quite simple, being mathematically only slightly more complex than the simplest dendrite growth systems. In the context of the present work, we also point out that electrodeposition systems nearly always involve the electric-field-mediated transport of charged molecules within the electrolyte solution. As we discuss below, the behavior of charged molecules in an electric field with large field gradients is significantly different from that of neutral molecules, and this leads to markedly different effects on the growth morphology.

Electric-field-induced transport of neutral particles has also been extensively studied for systems of aerosol particles, and has found widespread industrial application as a means to remove contaminants from air streams. Pattern formation in such systems has also been studied, and long filamentary structures are often observed [17]. Here again, in the context of the work reported here, the formation of patterns from aerosols involves complex surface processes that are not easily modeled, whereas the dendrite growth we are considering is governed primarily by simple surface tension.

As we reported previously [18], electrically enhanced diffusion in the present case results in a new instability mechanism in dendrite growth. If the electrical potential being applied to the dendrite is raised above a threshold value, the dendrite growth becomes unstable, and the tip velocity can no longer be limited by surface tension. Experimentally we found this leads to a new runaway growth regime, producing thin branchless needle crystals growing with much higher than normal tip velocities. We describe here in some detail a modified solvability theory, including electrically enhanced diffusion, which appears to describe the observed phenomenon. We also describe below how tip heating may be responsible for stabilizing the needle tip velocity in the fast growth regime.

\section{DENDRITE GROWTH THEORY}

To simplify the notation and treatment of the problem, we focus on a specific example of dendrite growth theory, namely growth via particle diffusion of solute molecules in a solvent gas, which best describes our experiments below. The solute concentration is assumed low, and the solute molecules are taken to be neutral with nonzero electric polarizability. The solvent molecules, however, are assumed neutral and unpolarizable. We also initially neglect the latent heat generated by the condensing molecules, since for low solute concentrations (small $v$ limit) this can be quickly removed by the solvent gas.

Thus the dendrite growth is governed by the diffusion equation which, in the presence of an external force $\vec{F}$, becomes the Smoluchowski equation [19]

$$
\frac{\partial c}{\partial t}=\vec{\nabla} \cdot(D \vec{\nabla} c-b c \vec{F})
$$

where $c(\vec{r})$ is the solute number density, $D$ is a scalar diffusion constant, and $b$ is the mobility of the solute molecules in the solvent. The particle mobility and diffusion constant are related via the Einstein relation, $b=D / k T$, so we can write

$$
\frac{\partial c}{\partial t}=D \vec{\nabla} \cdot(\vec{\nabla} c+c \vec{f})
$$

with $\vec{f}=-b \vec{F} / D=-\vec{F} / k T$. For electrically enhanced diffusion the applied force arises from an electrostatic potential, which is applied to the growing dendrite via an electrical connection far from the dendrite tip. We take the solute molecules to have polarizability $\alpha$, and assume the growing dendrite is a perfect conductor, which is reasonable since in our experiments the current flow induced by the applied potential is negligible. With these assumptions, the external force can be written as the gradient of a potential $\vec{f}=-\vec{\nabla}(\vec{p} \cdot \vec{E}) / k T$ $=-\vec{\nabla} \Phi$, where

$$
\Phi=\frac{\alpha}{k T}(\vec{E} \cdot \vec{E})
$$

and the electric field in turn is the gradient of the electrical potential $\vec{E}=-\vec{\nabla} \varphi$.

Ignoring interface kinetics, the continuity equation at the interface yields the normal component of the surface growth rate $v_{n}=\left(\hat{n} \cdot \vec{v}_{\text {surf }}\right)$ as

$$
v_{n}=\left.\frac{D}{c_{\text {solid }}} \hat{n} \cdot\left(\vec{\nabla}_{c}+c \vec{f}\right)\right|_{\text {surf }},
$$


where $c_{\text {solid }}$ is the solid number density and the right-hand side is evaluated at the solidification front [19].

\section{A. Spherical case}

It is instructive to solve the above in the simple case of a growing sphere in the limit $v \rightarrow 0$. Then $\partial c / \partial t \approx 0$ and the diffusion equation can be integrated to give

$$
c(r)=c(R)+\frac{B}{R}-\frac{B}{r}-\int_{R}^{r} f c d \hat{r}
$$

with

$$
B=R\left[\Delta c+\int_{R}^{\infty} f c d \hat{r}\right],
$$

where $R$ is the sphere radius and $\Delta c=c_{\infty}-c(R)>0$. This gives a growth velocity

$$
v=\frac{D c_{\text {sat }}}{R c_{\text {solid }}}\left[\Delta_{1}-\frac{d_{0}}{R}+c_{\text {sat }}^{-1} \int_{R}^{\infty} f c d \hat{r}\right],
$$

where $c(R)=c_{\text {sat }}\left(1+d_{0} / R\right)$ includes the Gibbs-Thomson effect, with $d_{0}=2 \beta / c_{\text {solid }} k T, \beta$ is the surface tension, $c_{\text {sat }}$ is the saturation number density, and $\Delta_{1}=\left(c_{\infty}-c_{\text {sat }}\right) / c_{\text {sat }}$.

For the electric force we have, from $f=-\partial \Phi / \partial r$ above, that

$$
f(r)=\frac{4 \alpha \varphi_{0}^{2} R^{2}}{k T r^{5}}
$$

where $\varphi_{0}$ is the external potential applied to the dendrite. Since this falls off very rapidly with $r$ in comparison with $c(r)$, the last term in Eq. (3) becomes

$$
c_{\mathrm{sat}}^{-1} \int_{R}^{\infty} f c d \hat{r} \approx\left(\frac{R_{0}}{R}\right)^{2},
$$

where $R_{0}=\left(\alpha \varphi_{0}^{2} / k T\right)^{1 / 2}$, giving

$$
v=\frac{D c_{\text {sat }}}{R c_{\text {solid }}}\left[\Delta_{1}-\frac{d_{0}}{R}+\left(\frac{R_{0}}{R}\right)^{2}\right] .
$$

Typical parameter values for the experiments described below are $T \approx-15^{\circ} \mathrm{C}, D \approx 2 \times 10^{-5} \mathrm{~m}^{2} \mathrm{sec}^{-1}$ for water molecules in air at one atmosphere, $c_{\text {sat }} / c_{\text {solid }} \approx 1.5 \times 10^{-6}$, and $\Delta_{1} \approx 0.5$. The measured surface tension is $\beta=0.109 \mathrm{~J} \mathrm{~m}^{-2}$ [20], giving $d_{0}=2.0 \mathrm{~nm}$ at $-15^{\circ} \mathrm{C}$, and the measured polarizability of water is [20]

$$
\begin{aligned}
\alpha & =\frac{\mu_{\mathrm{eff}}}{E}=\left(\mu_{0}^{2} / 3 k T\right)+\alpha^{\prime} \\
& \approx 3.4 \times 10^{-39} C^{2} m N^{-1}
\end{aligned}
$$

so

$$
R_{0}=\left(\frac{\alpha \varphi_{0}^{2}}{k T}\right)^{1 / 2} \approx(1 \mu \mathrm{m})\left(\frac{\varphi_{0}}{1000 \mathrm{~V}}\right)
$$

At this point we can make the century-old observation that electrically enhanced diffusion leads to an instability in the growth of droplets formed from a vapor of polarizable molecules, which is responsible for the visualization of charged-particle tracks in a cloud chamber. For uncharged particles, the Gibbs-Thomson effect provides that droplets with $R<R_{\text {crit }}=d_{0} / \Delta_{1}$ have a growth velocity $v<0$, and thus will evaporate. However, since the last term in Eq. (4) increases faster than $R^{-1}$ with decreasing $R$, sufficiently charged droplets will experience runaway growth for all $R$. That this very same simple effect produces an analogous runaway instability in dendrite growth was only recently recognized [18].

One might think that this dendrite growth instability would also be present in the dendritic and fractal growth of electrochemical systems, which have been extensively studied. Electrochemical deposition is a significantly more complex process, which has produced a rich phenomenology; nevertheless, the same basic physical mechanismselectrically enhanced diffusion and surface tension-are often dominant. In electrochemical deposition, however, the solute particles are charged, which significantly changes the nature of the electrically enhanced diffusion. For the spherical case with solute particles possessing charge $q$, the external force is $\vec{F}=q \vec{E}$, which yields the growth velocity

$$
v=\frac{D c_{\text {sat }}}{R c_{\text {solid }}}\left[\Delta_{1}-\frac{d_{0}}{R}-\frac{q \varphi_{0}}{k T}\right]
$$

Since the additional term has no $R$ dependence, surface tension can still stabilize the growth, in contrast to the neutral case. We cannot definitely conclude that there will not be additional growth instabilities in charged systems; however, we can conclude that growth from neutral polarizable molecules may exhibit phenomena that have not been seen in electrochemical systems.

\section{B. The modified Ivantsov solution}

Since the electrical force added to the diffusion equation does not have a particularly simple form in parabolic coordinates, it appears to be impossible to write down an exact solution analogous to the Ivantsov solution for growing dendrites in the limit of zero surface tension. Numerical techniques can be used to address this problem [21], but we find that we can obtain an approximate analytic expression that appears to contain all the essential physics. Because $c_{\text {sat }} / c_{\text {solid }} \ll 1$, the dendrite tip velocity is very slow and the diffusion length $l_{D}=2 D / v$ is much larger than the typical size of an experimental apparatus. In this slow growth limit we can take $\partial c / \partial t=0$ in the diffusion equation. Assuming a parabolic crystal surface at potential $\varphi_{0}$, the electric potential is simply

$$
\varphi=A \ln (\eta / R)+\varphi_{0}
$$

in parabolic coordinates, where $A=-\varphi_{0} / \ln \left(\eta_{\infty} / R\right)$ and we assume $\varphi=0$ on the far boundary at $\eta=\eta_{\infty}$. The external force potential then becomes

$$
\Phi=\frac{\alpha}{k T}\left(\frac{4 \eta}{\eta+\xi}\right) \frac{A^{2}}{\eta^{2}} .
$$


Since this falls off rapidly away from the dendrite tip, we take the diffusion equation to be $\nabla^{2}\left(c-c_{\text {sat }} \Phi\right)=0$, and neglect the $\xi$ dependence of $\Phi$, taking $\Phi \approx \Phi(\eta)$ $=4 \alpha A^{2} / k T \eta^{2}$. We then have

$$
\begin{gathered}
c(\eta)-c_{\mathrm{sat}} \Phi(\eta)=B \ln (\eta / R)+c_{\mathrm{sat}}-c_{\mathrm{sat}} \Phi_{\mathrm{surf}}, \\
B=\frac{\Delta c-c_{\mathrm{sat}} \Delta \Phi}{\ln \left(\eta_{\infty} / R\right)},
\end{gathered}
$$

which yields the tip velocity

$$
v=\frac{2 D}{R \ln \left(\eta_{\infty} / R\right)} \frac{c_{\text {sat }}}{c_{\text {solid }}}\left(\Delta_{1}+\frac{R_{\text {elec }}^{2}}{R^{2}}\right)
$$

in the absence of surface tension, where

$$
R_{\mathrm{elec}}^{2}=\frac{4 \alpha A^{2}}{k T}=\frac{4 \alpha \varphi_{0}^{2}}{k T \ln ^{2}\left(\eta_{\infty} / R\right)} .
$$

For $R_{\text {elec }}=0$ this becomes the slow-growth limit of the Ivantsov solution if we identify $\eta_{\infty} \approx l_{D}$.

\section{Solvability theory}

We next need to include surface tension (capillarity) in the dendrite growth theory, in order to stabilize the tip growth and select a unique tip radius and velocity. For this we work in the slow-growth limit and follow the solvability theory described in [9], extending it to include the effects of electrically enhanced diffusion. Writing the diffusion equation as $\nabla^{2} c=c_{\text {sat }} \nabla^{2} \Phi$, we can treat the right-hand side as a source term, and in analogy with electrostatics we use Green's theorem to rewrite the differential equation as an integral equation [22],

$c(\vec{x})=\frac{c_{\text {sat }}}{4 \pi} \int \frac{\nabla^{2} \Phi}{X} d V^{\prime}+\frac{1}{4 \pi} \oint\left[\frac{1}{X} \frac{\partial c}{\partial n^{\prime}}-c \frac{\partial}{\partial n^{\prime}}\left(\frac{1}{X}\right)\right] d A^{\prime}$,

where $X=\left|\vec{x}-\vec{x}^{\prime}\right|$, and the volume and surface integrals are over the region external to the growing crystal. The surface integrals over the far boundary reduce simply to $c_{\infty}$, and the surface gradient of $c$ is equal to $\partial c / \partial n^{\prime}=\left(c_{\text {solid }} /\right.$ $D)\left(\hat{n} \cdot \vec{v}_{\text {surf }}\right)$. Assuming shape-preserving growth with a velocity $\vec{v}=v \hat{z}$, and an approximately parabolic shape with tip radius of curvature $R$, this becomes

$$
\begin{aligned}
c(\vec{x})= & c_{\infty}+\frac{c_{\text {sat }}}{4 \pi} \int \frac{\nabla^{2} \Phi}{X} d V^{\prime}-\frac{1}{4 \pi} \frac{c_{\text {solid }}}{D} \\
& \times \oint\left[\frac{1}{X}(\hat{n} \cdot \hat{z})\right] d A^{\prime}+\frac{1}{4 \pi} \oint\left[c \frac{\partial}{\partial n^{\prime}}\left(\frac{1}{X}\right)\right] d A^{\prime},
\end{aligned}
$$

where the surface integrals are now over only the crystal surface. Here the signs have changed so $\hat{n}$ now points out from the crystal surface. Placing $\vec{x}$ on the crystal surface, with $c_{\text {surf }}=c_{\text {sat }}+c_{\text {sat }} \kappa d_{0}$, where $\kappa$ is the inverse mean radius of curvature, and performing the volume integral over a paraboloid-shaped crystal yields

$$
\begin{aligned}
c_{\text {sat }} & d_{0}\left\{\kappa-\frac{1}{4 \pi} \oint\left[\kappa\left(x^{\prime}\right)\left(\hat{n}^{\prime} \cdot \vec{\nabla}\left(\frac{1}{X}\right)\right)\right] d A^{\prime}\right\} \\
& =\Delta c+K_{0} c_{\text {sat }} \frac{R_{\text {elec }}^{2}}{R^{2}}-\frac{1}{4 \pi} \frac{c_{\text {solid }} v}{D} \oint\left[\frac{1}{X}(\hat{n} \cdot \hat{z})\right] d A^{\prime},
\end{aligned}
$$

where $K_{0}$ is a numerical factor of order unity, the value of which depends weakly on the detailed form of the dendrite solution. Expressing $\Delta c$ as

$$
\Delta c=\frac{1}{4 \pi} \frac{c_{\text {solid }}}{D} \oint\left[\frac{1}{X_{0}}\left(\hat{n}_{0} \cdot \hat{z}\right)\right] d A_{0}^{\prime},
$$

which is the $d_{0}=0$ expression [9], then yields

$$
\begin{aligned}
c_{\mathrm{sat}} d_{0} \kappa^{\prime}= & K_{0} c_{\text {sat }} \frac{R_{\text {elec }}^{2}}{R^{2}}+\frac{1}{4 \pi} \frac{c_{\text {solid }}}{D} \\
& \times\left\{\oint\left[\frac{1}{X_{0}}\left(\hat{n}_{0} \cdot \hat{z}\right)\right] d A_{0}^{\prime}-\oint\left[\frac{1}{X}(\hat{n} \cdot \hat{z})\right] d A^{\prime}\right\},
\end{aligned}
$$

where $\kappa^{\prime}$ is equal to the quantity in curly brackets in Eq. (7). Rewriting this in dimensionless length coordinates, $\widetilde{X}$ $=X / R$, etc., gives

$$
\begin{aligned}
\sigma \widetilde{\kappa}^{\prime}= & K_{0} \widetilde{R}_{\text {elec }}^{2} \frac{2 D}{v R} \frac{c_{\text {sat }}}{c_{\text {solid }}}+\frac{1}{2 \pi} \\
& \times\left\{\oint\left[\frac{1}{\widetilde{X_{0}}}\left(\widehat{n_{0}} \cdot \hat{z}\right)\right] \widetilde{d A}_{0}^{\prime}-\oint\left[\frac{1}{\widetilde{X}}(\hat{n} \cdot \hat{z})\right] \widetilde{d A}^{\prime}\right\},
\end{aligned}
$$

where

$$
\sigma=\frac{2 d_{0} D}{v R^{2}} \frac{c_{\text {sat }}}{c_{\text {solid }}}
$$

Comparing this with normal solvability theory, for which $R_{\text {elec }}=0$, and substituting in the modified Ivantsov result for $v R$, we can then write

$$
\sigma \approx \sigma_{0}+K \frac{R_{\mathrm{elec}}^{2}}{R^{2}}\left(\Delta_{1}+\frac{R_{\mathrm{elec}}^{2}}{R^{2}}\right)^{-1}
$$

where $\sigma_{0}$ can be recognized as the solvability parameter for $R_{\text {elec }}=0$, namely in the absence of electrically enhanced diffusion, and $K$ is a positive constant.

It is well known that the analysis so far, while describing an essential scaling relation for dendrite growth, falls considerably short of solving the entire problem. In the $R_{\text {elec }}=0$ case, for example, further analysis is needed to show that $\sigma_{0} \rightarrow 0$ in the absence of an anisotropic surface tension, and that there is no stable shape-preserving growth in that limiting case [6-9]. We assume that our initial assumption of shape-preserving growth is valid, requiring some anisotropy in the surface tension to produce a nonzero value of $\sigma_{0}$. Much of the detailed physics arising from the addition of electrically enhanced diffusion has been bundled into the value of the numerical constant $K$, which is seen to depend on the detailed dendrite solution in an intimate way. A full computation to determine $K$ would be quite complex, no 
doubt requiring numerical evaluation. However, examination of the implications of the scaling relation (8) suggests that the precise value of $K$ (even taking $K \rightarrow 0$ ) has only a minor effect on the growth behavior, which is easily parametrized for comparison with observations.

Combining the solvability relation (8) and the modified Ivantsov relation (5) yields a simple quadratic equation for the dendrite tip radius

$$
R^{2}-R_{0} R+A R_{\text {elec }}^{2}=0,
$$

where $A=\left(\sigma_{0}+K\right) / \sigma_{0} \Delta_{1}$ and $R_{0}=d_{0} \ln \left(\eta_{\infty} / R\right) / \sigma_{0} \Delta_{1}$ is the normal selected dendrite tip radius when $R_{\text {elec }}=0$ [note that since $\eta_{\infty} \gg R, \ln \left(\eta_{\infty} / R\right)$ depends only very weakly on $\left.R\right]$. In our previous paper we assumed a constant solvability parameter, which is equivalent to taking $K=0$. The above more rigorous treatment makes a small change in the value of $A$, but yields qualitatively the same basic result as was found in [18].

For $R_{\text {elec }} \ll R_{0}$ this gives the tip radius $R \approx R_{0}(1$ $\left.-A R_{\text {elec }}^{2} / R_{0}^{2}\right)$ and tip velocity $v \approx v_{0}\left(1+A^{\prime} R_{\text {elec }}^{2} / R_{0}^{2}\right)$, where $A^{\prime}=A+1 / \Delta_{1}$. The radius decreases with increasing $R_{\text {elec }}$ until a limit is reached when $R_{\text {elec }}=R_{0} / 2 A^{1 / 2}$, at which point $R=R_{\min }=R_{0} / 2$ and $v=v_{\max }=2 v_{0}\left(2 \sigma_{0}+K\right) /\left(\sigma_{0}+K\right)$. The quadratic equation has no real roots for $R_{\text {elec }}>R_{0} / 2 A^{1 / 2}$, indicating that the above modified solvability theory cannot be used for large $R_{\text {elec }}$, or equivalently when $\varphi_{0}$ is above some threshold potential $\varphi_{\max }$.

The lack of real roots for large applied potentials is related to the familiar phenomenon of nucleation in a cloud chamber, as we found above from the spherically symmetric solution to the Smoluchowski equation. In the present case of dendrite growth, above a threshold potential we find that surface tension can no longer stabilize the tip radius. At this point the tip experiences runaway growth as $R \rightarrow 0$ under the influence of the Mullins-Sekerka instability (here enhanced by electric forces). The tip velocity thus increases until it is stabilized by some other mechanism.

\section{Stabilization of electric needle growth}

We refer to crystal growth with $\varphi>\varphi_{\max }$ as the "electric' needle growth regime, which is distinct from the normal growth regime in that the tip velocity is no longer stabilized by the Gibbs-Thomson effect of surface tension. Observations in this regime (see below) reveal a rapidly growing needle morphology, which again exhibits a shapepreserving growth. For this to occur we must have some new physical mechanism which stabilizes the tip velocity against the Mullens-Sekerka instability. Furthermore, this mechanism must turn on very rapidly with decreasing tip radius, more rapidly than the Gibbs-Thomson effect, in order to provide the necessary stabilizing effect in the electric growth regime.

We suggest that tip heating, arising from the latent heat generated by the condensing molecules, is a likely candidate for a stabilizing effect. The vapor pressure above the solid is seen from the Clausius-Clapeyron equation to increase exponentially with increasing temperature, as $p \sim \exp \left(-T_{0} / T\right)$, where $T_{0}$ is equal to the latent heat per molecule divided by the Boltzmann constant. Thus a small increase in $T$ with increasing tip velocity $v$ may be sufficient to stabilize the electric needle growth. Tip heating was ignored in the discussion above, which is valid for small $v$, but can become significant at the higher $v$ produced by electric growth, as is seen in using the following argument.

We consider the growth of a semi-infinite rod of constant radius $R$, where condensing molecules cause the rod to increase its length with velocity $v$. Heat is deposited at the growing end of the rod at a rate $\dot{Q}_{\text {input }}=\alpha \dot{M}$, where $\alpha$ is the latent heat of condensation, $\dot{M}=\pi R^{2} v \rho$ is the mass deposition rate, and $\rho$ is the solid density. We assume that this heat conducts along the rod for some distance $L$, and is then conducted into the surrounding solvent gas. Convection will also play a role in removing heat into the surrounding medium, but we will ignore its contribution for this approximate treatment. Conduction down the rod is approximately $\dot{Q}_{\text {rod }}$ $\approx \kappa_{\text {solid }}\left(\pi R^{2}\right) \Delta T / L$, where $\kappa_{\text {solid }}$ is the conductivity of the solid and $\Delta T=T_{\text {tip }}-T_{\text {ambient }}$ is the temperature difference between the end of the rod and the ambient medium. Conduction into the surrounding gas is approximately $\dot{Q}_{\text {solvent }}$ $\approx \kappa_{\text {solvent }} \pi L \Delta T / \ln \left(R_{\infty} / R\right) \approx \kappa_{\text {solvent }} L \Delta T$. Since in steady state we must have $\dot{Q}_{\text {input }}=\dot{Q}_{\text {rod }}=\dot{Q}_{\text {solvent }}$, this gives $\Delta T$ $\approx\left(\kappa_{\text {solid }} \kappa_{\text {solvent }}\right)^{1 / 2} \alpha \rho v R$. From Eq. (5) above we can see that $v R$ increases with decreasing $R$ as $v R \sim\left(R_{\text {elec }} / R\right)^{2}$ for large $R_{\text {elec }}$ in the electric growth regime. Thus we find a tip heating that is strongly dependent on $R$, which will act to stabilize the needle growth. As we discuss below, additional tip heating may result from ionization effects, if the electric fields at the tip become excessive.

\section{EXPERIMENTS WITH ICE DENDRITE GROWTH}

An experimental demonstration of this growth instability was realized by growing ice dendrites from water vapor $[18,23]$. In many regards water ice is not an ideal experimental system for these measurements, since it is known that surface kinetic effects are usually very important in ice crystal formation, resulting in strongly faceted crystal growth [24-26]. The above theoretical calculations, however, are based on standard solvability theory, which does not include any such surface kinetic effects. Furthermore, the surface structure of ice is very complex, being dominated by the dynamics of a thin quasiliquid layer [25], resulting in a rich crystal growth behavior as a function of temperature and supersaturation [26]. From the experimental side, however, water ice is very easy to work with, given its convenient freezing temperature, and the large polarizability of water molecules facilitates the electrical effects we wish to observe.

We find that we can greatly reduce the effects of surface kinetics by growing ice dendrites at a temperature near $-15^{\circ} \mathrm{C}$ (near the dendrite growth peak [26]) and at supersaturations $\Delta_{1} \geqslant 0.4$. In this region of parameter space the prism faces of the growing dendrites are rough, yielding dendrites with approximately parabolic tips. In this parabolic regime, the growth of the prism faces is described by the Hertz-Knudsen relation [27], and is no longer dominated by surface kinetics. In contrast, growth of the basal faces in this regime is still affected by surface kinetics, producing nearly flat two-dimensional dendrites. However, on top of the flat 


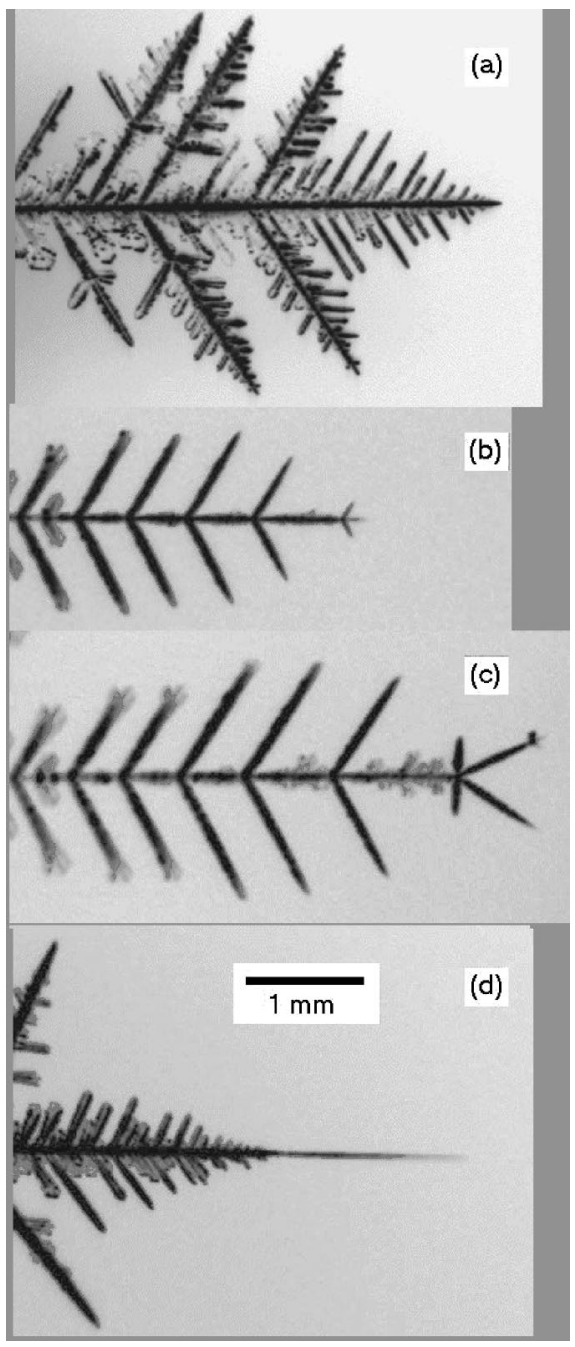

FIG. 1. Morphologies of ice crystal dendrites grown from supersaturated air at $-15^{\circ} \mathrm{C}$. All four images have the same scale. (a) Normal dendrite growth at $\Delta_{1} \approx 1$, with no applied electrical potential; (b) unusual growth at a potential of $2000 \mathrm{~V}$, with $\Delta_{1} \approx 1$, showing the supression and stabilization of sidebranching. The tip velocity and sidebranch spacing both increased as the tip advanced into surroundings with higher supersaturation. Note that in this morphology the sidebranches appear fully developed very near the tip region; (c) a continuation of the growth in (b), showing a common tip splitting instability that occurs above the threshold potential. Here there appears a sudden rotation of the crystal axes by $30^{\circ}$; (d) a dendrite grown at $\Delta_{1} \approx 0.5$, showing a smooth transition from normal growth to a rapidly growing needle morphology. The tip velocities before and after the transition are 5 and $70 \mu \mathrm{m} / \mathrm{sec}$, respectively.

dendrite plate we typically observe a ridgelike growth, which forms a "backbone" as the dendrite growth proceeds, as can be seen in Fig. 1. This backbone structure is also commonly seen in the growth of "sectored plate" ice crystals, and its formation is at present poorly understood [28]. Nevertheless, the backbone results in a dendrite tip structure which is not nearly as flat as the dendrite as a whole, and thus the approximation of a paraboloidal shape is a reasonably accurate one near the dendrite tip.

The experiments were performed in a vertical thermal diffusion chamber, measuring $15 \mathrm{~cm}$ in width and depth, and 32 $\mathrm{cm}$ in height. The chamber was of a triple-walled acrylic

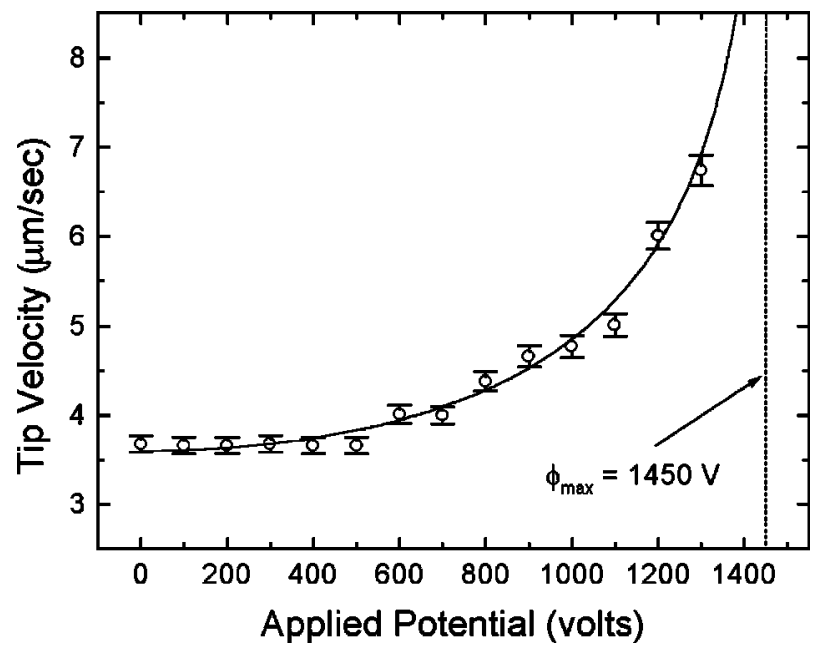

FIG. 2. Data points show measurements of the tip velocity of a single growing dendrite as a function of the applied electrical potential. The solid line is a fit to the points using the modified solvability theory described in the text. The two free parameters in the theory, $v_{0}$ and $\varphi_{\max }$, were adjusted in a least-squares fit to the data, which gave a best fit $\varphi_{\max }=1450 \mathrm{~V}$. This dendrite underwent a tip-splitting instability when the potential was raised from 1300 to $1400 \mathrm{~V}$

construction, being cooled at the base by a commercial chiller system. Both the top and bottom of the chamber were temperature regulated using an inner layer of thermoelectric coolers controlled by a servo mechanism [29]. The solvent gas in the chamber was ordinary laboratory air at one atmosphere of pressure, and water vapor was supplied at the top of the chamber via a water-soaked felt reservoir. We observed the crystal growth to be somewhat sensitive to vapor impurities in the air, particularly solvents from glue used in the chamber construction, so care was taken to minimize these effects. Imaging of the growing crystals was done from the side, using a standard video camera attached to one eye of a long-distance stereo microscope. Images were digitized using a video frame grabber attached to a personal computer, with software for time-lapse imaging. The images were all digitized at a resolution of $640 \times 480$ pixel resolution, with a typical scale of $10 \mu \mathrm{m} /$ pixel. Figure 1 shows several examples of dendrite images.

Ice dendrites were grown on a thin tungsten wire, through which an electrical potential was applied. A quantitative comparison with the above theory was realized by first establishing the growth of a single normal $\left(\varphi_{0}=0\right)$ dendrite, which had a tip velocity of typically $v \approx 3 \mu \mathrm{m} / \mathrm{sec}$. After achieving a stably growing dendrite, an electric potential was applied to the tungsten wire. The finite conductivity of ice and slow dendrite growth, along with the very low current flow [23], ensured that the dendrite possessed a nearly equipotential surface. Occasionally the applied potential resulted in dendrites with a large uniform sidebranch spacing, which is shown in Figs. 1(b) and 1(c). This behavior is rare and is currently unexplained.

More typically the dendritic growth remained qualitatively similar to that of normal growth at low potentials, with $v$ increasing with $\varphi_{0}$ up to a threshold potential. Figure 2 shows measurements of the tip velocity of a single growing dendrite, where $\varphi_{0}$ was increased in steps during the growth. 

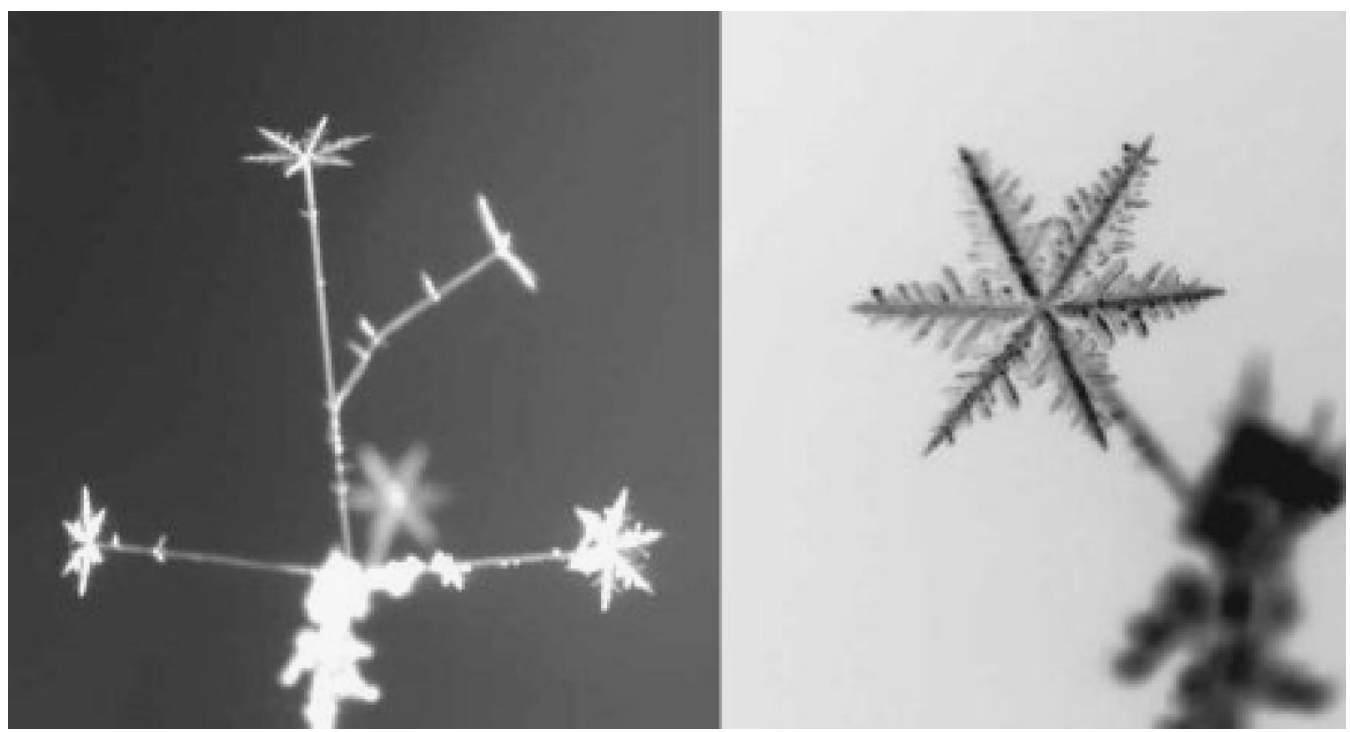

FIG. 3. Group of five ice crystal needles (left), grown along the [0001] axis at $-5{ }^{\circ} \mathrm{C}$ using an applied potential above threshold. After the needles grew to approximately $1 \mathrm{~cm}$ in length, the electric potential was turned off and the crystals were lowered to $-15^{\circ} \mathrm{C}$ in the diffusion chamber, which is the temperature at which platelike dendrites form. This resulted in the growth of normal single-crystal dendritic stars at the needle ends; one of these is shown as a negative image at right, which has a diameter of $2.4 \mathrm{~mm}$.

After each step in $\varphi_{0}$, the growth was allowed to stabilize to a constant tip velocity. We observe that $v$ increases slowly with increasing $\varphi_{0}$, until a threshold potential is reached, as is expected from the modified solvability theory above.

A comparison of the data and theory can be made by combining Eqs. (8) and (9) above into the approximate parametrized expression [18] $v \approx 4 v_{0}\left[1+\left(1-\varphi_{0}^{2} / \varphi_{\max }^{2}\right)^{1 / 2}\right]^{-2}$, where $v_{0}$ is the normal tip velocity. In Fig. 2 the two parameters $v_{0}$ and $\varphi_{\max }$ were adjusted via least-squares to best fit the measured points. This fit gave $\varphi_{\max }=1450 \mathrm{~V}$, which was in reasonable agreement with the observed threshold value. Unfortunately, a more quantitative comparison between theory and experiment is very difficult with this system, owing to the unusual characteristics of ice growth.

Above a threshold potential the enhanced dendrite growth behavior became unstable. If the potential was slowly raised above threshold with $\Delta_{1} \lesssim 0.6$, the dendrite tip would sometimes make a smooth transition to a rapid growth behavior, as shown in Fig. 1(d). This resulted in a thin, featureless needle-shaped crystal, with a diameter of $\sim 20-30 \mu \mathrm{m}$, moving at velocities typically $20-70 \mu \mathrm{m} / \mathrm{sec}$ along the original $a$-axis direction (although velocities as fast as $200 \mu \mathrm{m} / \mathrm{sec}$ were observed). The rapid "electric" needle velocities were observed to show considerable variation, which remains mysterious since it was not always simply correlated with the external growth conditions. A quantitative investigation of the details of electric needle growth is currently in progress.

Not surprisingly, given the complex surface structure of ice, we observed a variety of electrically induced growth behaviors [23]. If the potential was slowly raised at higher saturations $\left(0.6<\Delta_{1}<2\right)$, the dendrite tip often underwent the tip-splitting instability shown in Fig. 1(c). This resulted in a restructuring of the crystal at the dendrite tip, so that further growth occurred from an appended crystal whose [1 100$]$ axis was approximately collinear with the $a$ axis of the original crystal [18]. We are uncertain of the cause of this peculiar behavior, which may be related to electrofreezing phenomena [30], possibly augmented by tip heating effects.

It is conceivable that the mobility of water molecules on the growing crystal surface may be affected by the strong electric field gradients near the sharp dendrite tip [31], and thus may possibly be responsible in some way for the instability reported here. We believe, however, that such effects are minor in comparison to the enhanced diffusion described above. First of all, the applied surface fields, while substantial, are small compared to intrinsic crystal surface fields. And second, we observed that the main growth instability described here did not exhibit any dependence on the sign of the applied potential. This observation also allows us to effectively rule out ionization effects near the dendrite tip as playing any significant role in the growth dynamics during electrically enhanced normal growth. Electric needle growth may be affected by ionization effects, however, particularly if significant additional tip heating results. The applied potentials and tip radii in the electric growth regime are such that fields at the tip are near the breakdown field for air, so ionization effects may be significant.

If a potential value above threshold were suddenly applied at any saturation, the result was usually the copious production of thin needle crystals, similar to that shown in Fig. 1(d), which typically appeared from the sharp corners of faceted crystals. We observed electric needle growth along many different crystalline axes, which were determined by removing the applied potential and observing the subsequent crystal growth morphology. Near $-5{ }^{\circ} \mathrm{C}$ we could routinely grow ice needles along the [0001] axis, while at $-15^{\circ} \mathrm{C}$ growth

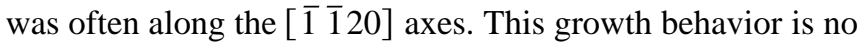
doubt related to the normal temperature dependence in ice crystal growth [26]. We also frequently observed electric needle growth along the [01̄0] axes [23], which is not currently understood. Furthermore, we often observed needle growth along seemingly random crystal orientations, suggesting that the electrically enhanced diffusion was overwhelming the otherwise dominant crystalline anisotropy. 


\section{DISCUSSION}

In summary, we have found a new type of dendrite growth instability, brought about by electrically enhanced diffusion of polar molecules in the presence of the dendrite tip. The nature of this external force provides that the presence of the dendrite itself affects the diffusion of material to its surface, adding a nontrivial nonlinear term to the diffusion equation. The principal result of theory and experiment is the existence of a threshold potential, above which dendrite growth can no longer be stabilized by surface tension effects. This instability is analogous to the droplet growth instability responsible for the visualization of charged particle tracks in cloud chambers.

We also note above that this threshold behavior is not expected to be present in electrochemical deposition systems, given the very different functional form of the electrically enhanced diffusion of ionic solute molecules. Thus, although dendrite growth and other growth instabilities have been seen in electrochemical deposition, the instability calculated above appears to be unique to growth from neutral polarizable molecules.

We note that the enhanced diffusion brought about by an applied potential is well understood at a fundamental level, and is straightforward to compute. Thus, the applied potential provides the experimenter with a convenient, continuously adjustable parameter with which to alter dendrite growth. Further studies on other polar systems with less complex surface properties in comparison to ice should provide new insights into pattern formation in diffusion-limited growth. Such studies may shed light on remaining problems in understanding the dependence of the stability parameter on crystalline anisotropy [11,32], and should also contribute to the general theory of morphological transitions during nonequilibrium growth [33].
In addition to its intrinsic interest relating to dendrite growth theory and phenomenology, this instability is particularly interesting because it results in the controlled growth of thin, featureless needle crystals, which can in principle be grown to any length. The growth dynamics of these needles is qualitatively different from needle crystals grown via other well-known techniques, for example via single screw dislocations at needle tips [34], or via the vapor-liquid-solid mechanism [35], and may be of some practical interest, particularly for the crystallization of large organic molecules, which can have substantial electric polarizabilities.

As a particular application, we find that the formation of long, thin, featureless electric needles is of some use in the study of ice crystal growth, which remains an extremely challenging case study because of strong three-dimensional surface kinetic effects, complicated by a quasiliquid layer. After first producing an electric needle with the desired crystalline orientation, one can then remove the electric potential and observe the subsequent growth of isolated single ice crystals, as is demonstrated in Fig. 3. By growing crystals at the end of long needles, the saturation is relatively unperturbed by the crystal support, allowing more detailed quantitative measurement of ice crystal growth dynamics. This is particularly true when investigating growth at high vapor supersaturations, where the perturbation from condensation on the crystal support can significantly interfere with the desired growth. This work also suggests that one may need to consider the effects of electrically enhanced diffusion when observing the growth of charged ice crystals held in an electrodynamic trap [36].

\section{ACKNOWLEDGMENTS}

The authors acknowledge support for V.M.T. by Mr. and Mrs. Downie D. Muir III.
[1] For reviews, see E. Ben-Jacob, Contemp. Phys. 38, 205 (1997); 34, 247 (1993); M. C. Cross and P. C. Hohenberg, Rev. Mod. Phys. 65, 851 (1993).

[2] H. Muller-Krumbhaar and W. Kurz, in Materials Science and Technology: A Comprehensive Treatment, edited by R. W. Cahn et al. (VCH, Weinheim, 1991), Vol. 5, Chap. 10.

[3] Y. Saito, Statistical Physics of Crystal Growth (World Scientific, Singapore, 1996).

[4] T. Vicsek, Fractal Growth Phenomena (World Scientific, Singapore, 1992).

[5] M. E. Glicksman and S. P. Marsh, in Handbook of Crystal Growth, edited by D. T. J. Hurle (North-Holland, Amsterdam, 1993), Vol. 1, Chap. 15.

[6] Y. Pomeau and M. Ben Amar, in Solids Far From Equilibrium, edited by C. Godreche (Cambridge University Press, Cambridge, England, 1992).

[7] J. S. Langer, in Chance and Matter: Les Houches Session XLVI edited by J. Souletie, J. Vannimenus, and R. Stora (Elsevier, New York, 1987), p. 629.

[8] H. Muller-Krumbhaar, in Morphology of Crystals, edited by I. Sunagawa (Terra Scientific, Tokyo, 1987).

[9] D. A. Kessler, J. Koplik, and H. Levine, Adv. Phys. 37, 255 (1988), and references therein.
[10] E. A. Brener, J. Cryst. Growth 166, 339 (1996); Phys. Rev. Lett. 71, 3653 (1993); A. Karma and W. J. Rappel, ibid. 77, 4050 (1996).

[11] A. Karma and W. J. Rappel, Phys. Rev. E 57, 4323 (1998); A. Karma and W. J. Rappel, J. Cryst. Growth 174, 54 (1997).

[12] U. Bisang and J. H. Bilgram, Phys. Rev. Lett. 75, 3898 (1995).

[13] G. P. Ivantsov, Dokl. Akad. Nauk SSSR 58, 1113 (1947).

[14] W. W. Mullins and R. F. Sekerka, J. Appl. Phys. 34, 323 (1963); J. S. Langer, Rev. Mod. Phys. 52, 1 (1980).

[15] Y. Sawada, A. Dougherty, and J. P. Gollub, Phys. Rev. Lett. 56, 1260 (1986); D. Grier et al., ibid. 56, 1264 (1986).

[16] J. R. de Bruyn, Phys. Rev. E 56, 3326 (1997), and references therein.

[17] For example, electrically induced filament formation from ice particles is described by L. K. Aksyutova et al., Sov. Tech. Phys. Lett. 11, 619 (1985) and L. F. Evans, J. Atmos. Sci. 30, 1657 (1973).

[18] K. G. Libbrecht and V. M. Tanusheva, Phys. Rev. Lett. 81, 176 (1998)

[19] S. Chandrasekhar, Rev. Mod. Phys. 15, 1 (1943).

[20] P. V. Hobbs, Ice Physics (Claredon, Oxford, 1974).

[21] Y. Saito, G. Goldbeck-Wood, and H. Muller-Krumbhaar, 
Phys. Rev. Lett. 58, 1541 (1987); Phys. Rev. A 38, 2148 (1988).

[22] J. D. Jackson, Classical Electrodynamics, 2nd ed. (Wiley, New York, 1975).

[23] J. T. Bartlett, A. P. van den Heuval, and B. J. Mason, Z. Angew. Math. Phys. 14, 599 (1963).

[24] Y. Saito, in Advances in the Understanding of Crystal Growth Mechanisms, edited by T. Nishinaga et al. (Elsevier, Amsterdam, 1997).

[25] Y. Furukawa and H. Nada, in Advances in the Understanding of Crystal Growth Mechanisms, edited by T. Nishinaga et al. (Elsevier, Amsterdam, 1997).

[26] Ice crystal growth from vapor is reviewed by T. Kobayashi and T. Kuroda, in Morphology of Crystals, Part B, edited by I. Sunagawa (Terra Scientific, Tokyo, 1987).

[27] P. Nozieres, in Solids Far From Equilibrium, edited by C. Godreche (Cambridge Univ. Press, Cambridge, England, 1992); E. Yokoyama, J. Cryst. Growth 128, 251 (1993); T.
Kuroda and R. Lacmann, ibid. 56, 189 (1982).

[28] F. C. Frank, Contemp. Phys. 23, 3 (1982).

[29] C. C. Bradley, J. Chen, and R. G. Hulet, Rev. Sci. Instrum. 61, 2097 (1990).

[30] I. M. Svishchev and P. G. Kusalik, J. Am. Chem. Soc. 118, 649 (1996); M. Gavish et al., Science 256, 815 (1992).

[31] C. R. Slaughterbeck et al., J. Vac. Sci. Technol. A 14, 1213 (1996).

[32] A. Dougherty and A. Gunawardana, Phys. Rev. E 50, 1349 (1994); M. Muschol, D. Liu, and H. Z. Cummins, Phys. Rev. A 46, 1038 (1992).

[33] O. Shochet et al., Physica A 187, 87 (1992).

[34] F. C. Frank, Discuss. Faraday Soc. 5, 48 (1949); W. Miao et al., J. Mater. Sci. 32, 1969 (1997)

[35] R. S. Wagner and W. C. Ellis, Appl. Phys. Lett. 4, 89 (1964); A. M. Morales and C. M. Lieber, Science 279, 208 (1998).

[36] N. J. Bacon et al., J. Geophys. Res. 103, 13763 (1998). 